\title{
12. \\ Sur le mouvement des fluides.
}

(Par Mr. A. F. Svanbery à Stockholm.)

(Présenté à l'Académie des sciences-de Stockholm 13 Nov, 1839.)

1. Je vais considérer dans ce mémoire le cas du mouvement des fluides, où tout est symétrique autour d'un axe donné quelconque. En effet il est aisé de prévoir, que la considération de ce cas devra simplifier les équations, d'où dépendent les lois du mouvement en général; et puisque dè plus on le rencontre très souvent. dans la nature, il parait pour celà même mériter l'attention particulière des géomètres. Quand par exemple un liquide s'écoule par une orifice circulaire pratiquée au milieu du fond d'un vase, dont la paroi est une surface quelconque de révolution avec un axe vertical, et qu'à l'état initial tout a été symétrique autour de cet axe, il devra aussi continuer de l'être pendant toute la durée de mouvement. Dans les tournans d'eau et dans les tourbillons la nature en fournit aussi d'exemples. Si pour les inconnues de la question on choisit outre la pression et la densité, qui pour les fluides élastiques est variable, $1^{\text {ment }}$ la vitesse d'une molecule parallèlement $\dot{a}$ l'axe, $2^{\text {ment }}$ la vitesse, avec laquelle elle s'approche ou s'éloigue de l'axe, $3^{\text {ment }}$ sa vitesse de rotation autour de l'axe, l'analyse nous donnera une loi bien rémarquable, quant à cette dernière, dont voici l'énoncé: si lon suit une molecule quelconque dans la courbe, qu'elle décrit pendant son mouvement, sa vitesse de rotation sera toujours en raison inverse des carrés de ses distances à l'axe.

Je suppose connues les suivantes formules fondamentales pour le mouvement des fluides:

$$
\begin{aligned}
& \text { 1. } \frac{1}{\varrho} \cdot \frac{d p}{d x}+\frac{d u}{d t}+u \frac{d u}{d x}+v \frac{d u}{d y}+v \frac{d u}{d z}=X, \\
& \text { 2. } \frac{1}{\varrho} \cdot \frac{d p}{d y}+\frac{d v}{d t}+u \frac{d v}{d x}+v \frac{d v}{d y}+w \frac{d v}{d z}=Y, \\
& \text { 3. } \frac{1}{\varrho} \cdot \frac{d p}{d z}+\frac{d w}{d t}+u \frac{d w}{d x}+v \frac{d w}{d y}+w \frac{d w}{d z}=Z, \\
& \text { 4. } \frac{d \varrho}{d t}+\frac{d(\varrho u)}{d x}+\frac{d(\varrho v)}{d y}+\frac{d(\varrho w)}{d z}=0,
\end{aligned}
$$


ou l'on a employé les notatios suivantes:

$x, y, z$ pour les coordonnées orthogonales d'une molecule quelconque de la masse fluide au bout du temps $t$,

$u, v, w$ les composantes parallèles aux axes coordonnés $x, y, z$ de la vitesse, dont la molecule est animée au bout du temps $t$, ou bien

$$
\text { 5. } \quad u=\frac{d x}{d t}, \quad v=\frac{d y}{d t}, \quad w=\frac{d z}{d t} ;
$$

$\boldsymbol{X}, \boldsymbol{Y}, \boldsymbol{Z}$ les composantes parallèles aux axes des $x, y, z$ de la force acceleratrice, qui agit sur la molecule à ce même instant, $p$ la pression et $\rho$ la densité.

2. Supposons maintenant, que pendant le mouvement tout soit symétrique autour de l'axe des $z$, et que pour celà nous appellerons l'axe du mouvement. Si au lieu des $x$ et $y$ on introduit les coordonnées polaires $r$ et $\theta$, de manière qu'on ait

6. $x=r \cos \theta, \quad y=r \sin \theta$,

et qu'on appelle $\mu$ la vitesse avec laquelle la molecule s'éloigne de l'axe, et $\gamma$ sa vitesse de rotation autour de lui, on aura

$$
\text { 7. } \mu=\frac{d r}{d t}, \quad \gamma=\frac{d \theta}{d t} \text {. }
$$

La différentiation des formules (6) donnera

$$
\text { 8. } u=\mu \cos \theta-\gamma r \sin \theta, \quad v=\mu \sin \theta+\gamma r \cos \theta \text {. }
$$

Mais puisque nous avons supposé, que pendant le mouvement tont soit symétrique autour de l'axe des $z$, il faut que $\mu, \gamma, w, p$ et $\rho$ ne varient pas, quand $\theta$ seul variera; d'où l'on conclut

9. $\frac{d \mu}{d \theta}=0, \quad \frac{d \gamma}{d \theta}=0, \quad \frac{d w}{d \theta}=0, \quad \frac{d p}{d \theta}=0, \quad \frac{d \varrho}{d \theta}=0$.

Les formules (6) différentiées, en regardant $y$ comme constant, donnent

$$
1=\cos \theta \frac{d r}{d x}-r \sin \theta \frac{d \theta}{d x}, \quad 0=\sin \theta \frac{d r}{d x}+r \cos \theta \frac{d \theta}{d x},
$$

d'où l'on tire

$$
\text { 10. } \frac{d r}{d x}=\cos \theta, \quad \frac{d \theta}{d x}=-\frac{\sin \theta}{r} ;
$$

de la même manière on trouve aussi

Mais on a

$$
\text { 11. } \frac{d r}{d y}=\sin \theta, \quad \frac{d \theta}{d y}=\frac{\cos \theta}{r} \text {. }
$$

12. $\frac{d u}{d x}=\frac{d u}{d r} \cdot \frac{d r}{d x}+\frac{d u}{d \theta} \cdot \frac{d \theta}{d x}, \quad \frac{d u}{d y}=\frac{d u}{d r} \cdot \frac{d r}{d y}+\frac{d u}{d \theta} \cdot \frac{d \theta}{d y}$, 
et en vertu des formules (8) et (9)

13. $\frac{d u}{d r}=\cos \theta \frac{d \mu}{d r}-r \sin \theta \frac{d \gamma}{d r}-\gamma \sin \theta, \quad \frac{d u}{d \theta}=-\mu \sin \theta-\gamma r \cos \theta$. Si dans les formules (12) on substitue les valeurs données par (10), (11) et (13) on trouve

$$
\begin{aligned}
& \frac{d u}{d x}=\cos \theta^{2} \frac{d \mu}{d r}-r \sin \theta \cos \theta \frac{d \gamma}{d r}+\frac{\mu}{r} \sin \theta^{2}, \\
& \frac{d u}{d y}=\sin \theta \cos \theta \frac{d \mu}{d r}-r \sin \theta^{2} \frac{d \gamma}{d r}-\frac{\mu}{r} \sin \theta \cos \theta-\gamma .
\end{aligned}
$$

De la même manière on obtient encore

$$
\begin{aligned}
& \frac{d v}{d x}=\sin \theta \cos \theta \frac{d \mu}{d r}+r \cos \theta^{2} \frac{d \gamma}{d r}-\frac{\mu}{r} \sin \theta \cos \theta+\gamma, \\
& \frac{d v}{d y}=\sin \theta^{2} \frac{d \mu}{d r}+r \sin \theta \cos \theta \frac{d \gamma}{d r}+\frac{\mu}{r} \cos \theta^{2}, \\
& \frac{d u}{d z}=\cos \theta \frac{d \mu}{d z}-r \sin \theta \frac{d \gamma}{d z}, \\
& \frac{d v}{d z}=\sin \theta \frac{d \mu}{d z}+r \cos \theta \frac{d \gamma}{d z}, \\
& \frac{d u}{d t}=\cos \theta \frac{d \mu}{d t}-r \sin \theta \frac{d \gamma}{d t}, \\
& \frac{d v}{d t}=\sin \theta \frac{d \mu}{d t}+r \cos \theta \frac{d \gamma}{d t}, \\
& \frac{d w}{d x}=\cos \theta \frac{d w}{d r}, \quad \frac{d w}{d y}=\sin \theta \frac{d w}{d r}, \\
& \frac{d p}{d x}=\cos \theta \frac{d p}{d r}, \quad \frac{d p}{d y}=\sin \theta \frac{d p}{d r}, \\
& \frac{d \varrho}{d x}=\cos \theta \frac{d \varrho}{d r}, \quad \frac{d \varrho}{d y}=\sin \theta \frac{d \varrho}{d r} .
\end{aligned}
$$

Substitutiant ces valeurs dans les formules (1) et (2) il vient

14.

$\left\{\frac{\cos \theta}{\rho} \cdot \frac{d p}{d r}+\cos \theta \frac{d \mu}{d t}-r \sin \theta \frac{d \gamma}{d t}+\mu \cos \theta \frac{d \mu}{d \phi}-\mu r \sin \theta \frac{d \gamma}{d r}\right.$ $\left\{-2 \mu \gamma \sin \theta-\gamma^{2} r \cos \theta+w \cos \theta \frac{d \mu}{d z}-w r \sin \theta \frac{d \gamma}{d z}=X\right.$,

15. $\left\{\begin{array}{l}\frac{\sin \theta}{\rho} \cdot \frac{d p}{d r}+\sin \theta \frac{d \mu}{d t}+r \cos \theta \frac{d \gamma}{d t}+\mu \sin \theta \frac{d \mu}{d r}+\mu r \sin \theta \frac{d \gamma}{d r} \\ +2 \mu \gamma \cos \theta-\gamma^{2} r \sin \theta+w \sin \theta \frac{d \mu}{d z}+w r \cos \theta \frac{d \gamma}{d z}=Y .\end{array}\right.$

Multipliant l'équation (14) par $\cos \theta$ et (15) par $\sin \theta$, puis les ajoutant, on trouve 
16. $\frac{1}{\rho} \cdot \frac{d \mu}{d r}+\frac{d \mu}{d t}+\mu \frac{d \mu}{d r}+w \frac{d \mu}{d z}-\gamma^{2} r=Y \sin \theta+X \cos \theta$. Si l'on multiplie l'équation (14) par $\sin \theta$ et (15) par $\cos \theta$, on aura en soustrayant la première de la seconde

17. $\quad r \frac{d \gamma}{d t}+r \mu \frac{d \gamma}{d r}+r w \frac{d \gamma}{d z}+2 \mu \gamma=Y \cos \theta-X \sin \theta$.

Supposons maintenant, que les seules forces, qui agissent sur chaque molecule, soient des attractions ou répulsions vers des centres fixes on mobiles, mais dont la résultante, à cause de la symétrie supposée autour de l'axe des $z$, doit avoir une direction qui passe par cet axe. Alors on aura

$$
\boldsymbol{X}: \boldsymbol{Y}=\boldsymbol{x}: y ; \quad \text { d'où } \quad \boldsymbol{Y} \boldsymbol{x}-\boldsymbol{X} \boldsymbol{y}=0,
$$

ou bien en substituant les valeurs de $x$ et $y$ données par les formules (6) 18. $\boldsymbol{Y} \cos \theta-\boldsymbol{X} \sin \theta=0$.

Si l'on fait

$$
\boldsymbol{Y} \sin \theta+\boldsymbol{X} \cos \theta=\boldsymbol{R},
$$

on trouvera en carrant

$$
\boldsymbol{Y}^{2} \sin \theta^{2}+2 \boldsymbol{Y} \boldsymbol{X} \sin \theta \cos \theta+\boldsymbol{X}^{2} \cos \theta^{2}=\boldsymbol{R}^{2}
$$

mais en vertu de (18) on a l'équation

$$
\boldsymbol{Y}^{2} \cos \theta^{2}-2 \boldsymbol{Y} \boldsymbol{X} \sin \theta \cos \theta+\boldsymbol{X}^{2} \sin \theta^{2}=0
$$

laquelle ajoutée à la précédante donne

$$
\boldsymbol{Y}^{2}+\boldsymbol{X}^{2}=\boldsymbol{R}^{2} ; \quad \text { d'où } \quad \boldsymbol{R}=\boldsymbol{V}\left(\boldsymbol{Y}^{2}+\boldsymbol{X}^{2}\right) \text {. }
$$

Cette expression de $\boldsymbol{R}$ fait voir, qu'après la substitution des valeurs de $x$ et $\boldsymbol{y}$ données par les formules (6) on obtiendra pour $\boldsymbol{R}$ une expression indépendante de $\theta$. Les équations (16) et (17) deviennent par conséquent

19. $\frac{1}{\rho} \cdot \frac{d p}{d r}+\frac{d \mu}{d t}+\mu \frac{d \mu}{d r}+w \frac{d \mu}{d z}-\gamma^{2} r=R$,

20. $\frac{d \gamma}{d t}+\mu \frac{d \gamma}{d r}+w \frac{d \gamma}{d z}+\frac{2 \mu}{r} \gamma=0$.

Les équations (3) et (4) donnent

$$
\begin{aligned}
\text { 21. } \frac{1}{\varrho} \cdot \frac{d p}{d z}+\frac{d w}{d t}+\mu \frac{d w}{d r}+w \frac{d w}{d z}=Z, \\
\text { 22. } \frac{d \varrho}{d t}+\frac{d(\varrho \mu)}{d r}+\frac{\rho \mu}{r}+\frac{d(\varrho w)}{d z}=0,
\end{aligned}
$$

dont la dernière peut aussi se mettre sous la forme suivante:

$$
\text { 23. } \frac{d \varrho}{d t}+\frac{1}{r} \cdot \frac{d(\varrho r \mu)}{d r}+\frac{d(\varrho w)}{d z}=0 \text {. }
$$


3. Le problème du mouvement que nous avons considéré, se trouve donc réduit à la solution des quatre équations données dans le numero précédent. $r, z$ et $t \mathrm{y}$ entrent comme variables indépendantes, c'est- $\dot{\mathrm{a}}-\mathrm{dire}$, ils n'appartienuent pas exclusivement à une même molécule de la masse fluide. Si au contraire on veut suivre une molécule quelconque dans la trajectoire qu'elle décrit pendant son nouvement, il faut considérer $r$ et $z$ comme fonctions de $t$. Désignons dans cette dernière supposition par $\frac{d \cdot \gamma}{d t}$ le coëfficient différential de $\gamma$ par rapport à $t$; nous aurons

$$
\frac{d \cdot \gamma}{d t}=\frac{d \gamma}{d t}+\frac{d \gamma}{d r} \cdot \frac{d r}{d t}+\frac{d \gamma}{d z} \cdot \frac{d z}{d t},
$$

ou, ce qui est le même,

$$
\frac{d \cdot \gamma}{d t}=\frac{d \gamma}{d t}+\mu \frac{d \gamma}{d r}+w \frac{d \gamma}{d z} .
$$

L'équation (20) pourra done s'écrire ainsi :

$$
\frac{d \cdot \gamma}{d t}+\frac{2 \gamma}{r} \cdot \frac{d r}{d t}=0
$$

et cela multiplié par $r^{2} d t$ donne

$$
r^{2} d \cdot \gamma+2 \gamma r d r=0,
$$

dont l'intégrale est

$$
r^{2} y=a,
$$

d'où enfin

$$
\text { 24. } \gamma=\frac{a}{r^{2}} \text {. }
$$

La constante $a$ peut en général varier d'une molécule à l'autre; mais pour chaque molécule séparément l'équation (24) donne la loi suivante, fort remarquable, savoir, que la vilesse de rotation la molécule dans divers points de sa trajectoire sera toujours en raison inverse des carrés des distances à l'axe du mouvement.

De l'équation (24) suit, que si les molécules pendant leur mouvement sont forcées de s'approcher de l'axe, leur vitesse de rotation ira toujours en croissant; ce résultat est en effet confirmé par l'expérience dans les tournans d'eau et dans les tourbillons. Si à l'état initial a a été $=0$ pour toutes les molécules, alors, et dans ce cas seulement, $\gamma$ sera constamment nul pendant toute la durée du mouvement.

4. En général, si à l'état initial on a communiqué à la masse fluide un mouvement de rotation tel, que $a$ soil constant pour toutes les molécules, 
il est évident que l'équation (24) devra subsister même en régardant $r, z$ et $t$ comme variables indépendantes. Nous supposerons celà, et pour abréger nous ferous

$$
\text { 25. } \int \frac{d p}{\varrho}=\boldsymbol{P} \text {. }
$$

De plus puisque nous avons supposé, que $\boldsymbol{R}$ et $\boldsymbol{Z}$ proviennent seulement des attractions on répulsions vers̀s des centres fixes ou mobiles, situés sur l'axe même des $z$, il faut qu'on ait

$$
\text { 26. } \quad R d r+\mathbb{Z} d z=d S \text {; }
$$

d'où

$$
R=\frac{d S}{d r}, \quad Z=\frac{d S}{d z}
$$

$S$ étant en général une fonction de $r, z$ et $t$, mais qui dans l'équation (26) est différentié seulement par rapport à $r$ et $z$. Les équations (19) et (21) peuvent donc s'écrire ainsi :

$$
\begin{aligned}
& \text { 27. } \frac{d P}{d r}+\frac{d \mu}{d t}+\mu \frac{d \mu}{d r}+w \frac{d \mu}{d z}-\frac{\alpha^{3}}{r^{3}}=\frac{d S}{d r}, \\
& \text { 28. } \frac{d P}{d z}+\frac{d w}{d t}+\mu \frac{d \mu}{d r}+w \frac{d \mu}{d z}=\frac{d S}{d z} .
\end{aligned}
$$

Pour éliminer $\boldsymbol{P}$, différentions l'équation (27) par rapport $\dot{a} z$ et (28) par rapport à $r$; puis rétranchons la seconde de la première. L'équation, qui en résultera, peut se mettre sous la forme suivante:

$$
\begin{aligned}
& \frac{d\left(\frac{d \mu}{d z}-\frac{d w}{d r}\right)}{d t}+\mu \frac{d\left(\frac{d \mu}{d z}-\frac{d w}{d r}\right)}{d r}+w \frac{d\left(\frac{d \mu}{d z}-\frac{d w}{d r}\right)}{d z} \\
& +\left(\frac{d \mu}{d r}+\frac{d w}{d z}\right)\left(\frac{d \mu}{d z}-\frac{d w}{d r}\right)=0 .
\end{aligned}
$$

Faisons pour abréger

$$
\frac{d \mu}{d z}-\frac{d w}{d r}=\Theta
$$

l'équation précédente donnera

29. $\frac{d \Theta}{d t}+\mu \frac{d \Theta}{d r}+w \frac{d \Theta}{d z}+\left(\frac{d \mu}{d r}+\frac{d w}{d z}\right) \Theta=0$.

Si nous désignons, comme nous l'avons fait précédemment, par $\frac{d . U}{d t}$ le coëfficient diffẻrentiel d'une fonction quelconque $U$ par rapport à $t$, en y regardant $r$ et $z$ aussi comme fonctions de $t$, nous aurons en général

$$
\frac{d U}{d t}+\mu \frac{d U}{d r}+\omega \frac{d U}{d z}=\frac{d . U}{d t},
$$


et par là l'équation (29) se changera en

$$
\text { 30. } \frac{d \cdot \Theta}{d t}+\left(\frac{d \mu}{d r}+\frac{d w}{d z}\right) \Theta=0 \text {. }
$$

L'équation (22) donne

ou bien

$$
\frac{d \varrho}{d t}+\mu \frac{d \varrho}{d r}+w \frac{d \varrho}{d z}+\varrho\left(\frac{d \mu}{d r}+\frac{d w}{d z}+\frac{\mu}{r}\right)=0,
$$

Delà on tire

$$
\frac{d . \varrho}{d t}+\rho\left(\frac{d \mu}{d r}+\frac{d w}{d z}+\frac{d r}{r d t}\right)=0 .
$$

par où l'équation (30) deviendra

$$
\frac{d \mu}{d r}+\frac{d w}{d z}=-\frac{d \cdot \varrho}{\varrho d t}-\frac{d r}{r d t}
$$

$$
\frac{d \cdot \Theta}{d t}=\Theta\left(\frac{d \cdot \varrho}{\varrho d t}+\frac{d r}{r d t}\right)
$$

laquelle multipliée par $\frac{d t}{\Theta}$ devient intégrable et donne

$$
\log \Theta=\log b \rho r,
$$

d'où nous obtenons enfin, en remettant pour $\Theta$ sa valeur,

$$
\text { 31. } \frac{d \mu}{d z}-\frac{d w}{d r}=b \rho r \text {. }
$$

La constante $\boldsymbol{b}$ est indépendante de la situation continuellement changeante de chaque molecule, mais qui généralement n'est pas la mème pour

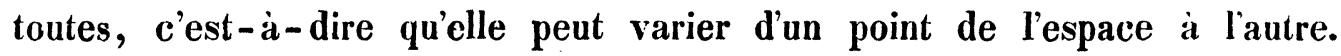
Sa valeur se déterminera d'après la valeur connue de $\Theta \dot{a}$ une époque quelconque donnée. Si à cette époque la valeur de $\Theta$ pour tous les divers points de la masse fluide est telle, qu'on obtient $\boldsymbol{b}$ constant pour toutes les molecules, il s'ensuit que l'équation (31) devra subsister pendant toute la durée du mouvement, même en regardant $r, z$ et $t$ comme des variables indépendantes. Si p. ex. à une époque quelconque on avoit

$$
\text { 32. } \frac{d \mu}{d z}-\frac{d w}{d r}=0
$$

pour tous les points du fluide, il s'ensuivroit $b=0$ dans l'équation (31), et par conséquent l'égalité (32) devroit subsister au bout d'un temps quelconque pour tous les points du fluide. Mais l'équation (32) est. celle de condition, pour que $\mu d r+w d z$ soit la différentielle complète d'une fonction quelconque $\phi$ par rapport $\dot{a} \boldsymbol{r}$ et $z$. Si donc on fait

33. $\mu d r+w d z=d \Phi$, 
ce qui donne

$$
\mu=\frac{d \varphi}{d r}, \quad w=\frac{d \varphi}{d z},
$$

et qu'on substitue ces valeurs dans les équations (27) et (28), il viendra

$$
\begin{aligned}
& \frac{d P}{d r}+\frac{d^{2} \varphi}{d r d t}+\frac{d \varphi}{d r} \cdot \frac{d^{2} \varphi}{d r^{2}}+\frac{d \varphi}{d z} \cdot \frac{d^{2} \varphi}{d r d z}-\frac{a^{2}}{r^{3}}=\frac{d S}{d r}, \\
& \frac{d P}{d z}+\frac{d^{2} \varphi}{d z d t}+\frac{d \varphi}{d r} \cdot \frac{d^{2} \varphi}{d r d z}+\frac{d \varphi}{d z} \cdot \frac{d^{2} \varphi}{d z^{2}}=\frac{d S}{d z}
\end{aligned}
$$

Ces équations donnent, si on les ajoute après avoir multiplié la première par $d r$ et la seconde par $d \approx$,

$$
d \boldsymbol{P}+d \cdot \frac{d \varphi}{d t}+\frac{1}{2} d \cdot\left[\left(\frac{d \varphi}{d r}\right)^{2}+\left(\frac{d \varphi}{d z}\right)^{2}\right]-a^{2} \frac{d r}{r^{3}}=d S,
$$

et l'intégration de cette équation donne

$$
\text { 34. } \quad \boldsymbol{P}+\frac{d \varphi}{d t}+\frac{1}{2}\left[\left(\frac{d \varphi}{d r}\right)^{2}+\left(\frac{d \varphi}{d z}\right)^{2}\right]-\frac{a^{2}}{2 r^{2}}=S \text {. }
$$

On pourroit y ajouter une fonction arbitraire de $t$, puisque dans l'intégration cette variable est régardée comme constante; mais comme cette fonction pent être censée renfermée dans la valeur de $\frac{d \varphi}{d t}$, on peut, sans déroger à la généralité de l'équation précédente, se dispenser de l'y ajouter. L'équation (22) donne

$$
\text { 35. } \frac{d \varrho}{d t}+\frac{d\left(\varrho \frac{d \varphi}{d r}\right)}{d r}+\frac{d\left(\rho \frac{d \varphi}{d z}\right)}{d z}+\frac{\varrho}{r} \cdot \frac{d \varphi}{d r}=0 .
$$

5. Supposons qu'il s'agit d'un fluide incompressible et que la pésanteur soit la seule force qui agit sur ses molécules. Alors $\rho$ est constant et on peut le supposer égal à l'unité; l'équation (25) donne par conséquent $\boldsymbol{P}=p$, et si l'on prend les $\boldsymbol{z}$ positives daus une direction contraire à celle de la pésanteur, on aura

$$
\boldsymbol{X}=0, \quad \boldsymbol{Y}=0, \quad \boldsymbol{Z}=-\boldsymbol{g},
$$

$g$ désignant la vitesse d'un corps pésant après une seconde de chùte libre. L'équation (26) donne

$$
S=-g z .
$$

Supposons que le mouvement ait comnencé du repos, nous aurons $a=0$ pour toutes les molécules, d'où l'on conclut en générạl $\gamma=0$. Puisque de plus $_{a} \dot{a}$ ce moment $\mu=0$ et $w=0, \mu d r+w d z$ est alors une différentielle complète, et par conséquent il continuera de l'être pendant toute la 
12. Svanberg, sur le monvement des Auides.

durée du mouvement. Les équations (34) et (35) donnent pour ce cas

$$
\text { 36. } \begin{aligned}
& p+\frac{d \varphi}{d t}+\frac{1}{2}\left\{\left(\frac{d \varphi}{d r}\right)^{2}+\left(\frac{d \varphi}{d z}\right)^{2}\right\}=-g z, \\
& \text { 37. } \quad \frac{d^{2} \varphi}{d r^{2}}+\frac{1}{r} \cdot \frac{d \varphi}{d r}+\frac{d^{2} \varphi}{d z^{2}}=0 .
\end{aligned}
$$

De ces deux équations dépendent les lois de l'écoulement d'un liquide par une orifice circulaire, pratiquée au milieu du fond d'un vase, dont la paroi soit une surface quelconque de revolution autour d'un axe vertical, le mouvement ayant commencé du repos, de manière qu'il n'y ait pas de mouvement tournoyant.

6. Puisque dans le cas du mouvement, que nous venons de considérer, $r$ et $\approx$ décroissent tandis que le temps croit, il est évident que $\mu$ et $w$ devront être négatives. Désignons par $\boldsymbol{r}^{\prime}$ la valeur de $\boldsymbol{r}$ pour l'orifice, et par $\boldsymbol{R}$ pour la paroi; $\boldsymbol{R}$ sera en général une fonction de $z$. Or soit que la surface du niveau s'abaisse, ou qu'elle ne varie pas, si l'on appelle $q$ le volume du liquide sorti du vase au bout du temps $t$, sa différentielle sera égale au volume du liquide, qui traverse un plan quelconque perpendiculaire à l'axe pendant l'iustant $d t$. Si donc dans un tel plan nous considérons un anneau circulaire compris entre les deux rayous $r$ et $r+d r$, son aire sera égale à $2 \pi r d r$, laquelle multipliée par $-w d t$ donne le volume du liquide, qui pendant l'instant $d t$ l'a traversé. En prenant la somme de ces produits depuis $r=0$ jusqu'à $r=R$, il vient

$$
\text { 38. } d q=-2 \pi d t \int_{0}^{R} w \cdot r d r \text {. }
$$

Cette équation devant subsister, quel que soit $z$, il faut que la différence partielle du second membre par rapport à cette variable soit égale à zéro; ce qui donne

$$
\frac{d \cdot \int_{0}^{R} w \cdot r d r}{d z}=0,
$$

ou bien, si l'on se rappelle que $\boldsymbol{R}$ est lui-même une fonction de $z$,

$$
\int_{0}^{R} \frac{d w}{d z} \cdot r d r+R w_{R} \frac{d R}{d z}=0
$$

$w_{R}$ désignant la valeur de $w$ pour $r=R$. Mais l'équation (23) donne, en faisant $\rho=1$,

39. $\quad \frac{d(r \mu)}{d r}+r \frac{d v}{d z}=0$ : 
l'équation précédante pourra donc s'écrire comme suit:

$$
\boldsymbol{R} w_{R} \frac{d R}{d z}-\int_{0}^{R} \frac{d(r \mu)}{d r} d r=0 .
$$

Lintégration étant effectuée entre les limites données, on obtient après avoir divisé par $\boldsymbol{R}$,

$$
\text { 40. } \quad w_{R} \frac{d \boldsymbol{R}}{d z}-\mu_{R}=0 ;
$$

$\mu_{R}$ étant la valeur de $\mu \dot{a}$ la limite $r=R$. L'équation (40) est en effet la condition necessaire pour que les mêmes particules du liquide soient toujours contiguës à la paroi. On a coutume de donner cette équation comme le résultat d'une supposition particulière; mais nous venońs de voir, qu'elle est une conséquence nécessaire de la continuité de la masse liquide.

7. Soit $z=0$ l'équation du fond du vase et $z=z_{1}$ l'équation de la surface supérieure: $z_{1}$ sera en général fonction de $r$ et $t$. D'abord il est évident, que les molecules voisines au fond du vase ne pourront avoir qu'un mouvement horizontal, et par conséquent qu'on aura $w=0$ pour $s=0$ et $r>r^{\prime}$.

Imaginons maintenant un cylindre circulaire, dont le rayon $r$ soit $>\boldsymbol{r}^{\prime}$ et dont l'axe coïncide avec celui du mouvement. Si l'on appelle $A$ la différence des volumes du liquide, qui aux temps $t$ et $t+d t$ sont contenus dans ce cylindre, et $\boldsymbol{B}$ le volume du liquide, qui dans le temps $d t$ a traversé sa surface, on aura, quel que soit d'ailleurs $\boldsymbol{r}$,

$$
\boldsymbol{d} \boldsymbol{q}=\boldsymbol{A}+\boldsymbol{B} \text {. }
$$

Or l'abaissement du niveau dans le temps $d t$ étant égal à $-\frac{d \approx_{1}}{d t} d t$, on trouvera de la même manière que dans le numero précédent,

$$
A=-2 \pi d t \int_{0}^{r} \frac{d z_{1}}{d t} r d r
$$

Pour obtenir $B$ il faut considérer un élément annullaire dont le rayon soit $r$ et la hauteur $d z$; l'aire de cet élément sera égale à $2 \pi r d z$. Si on la multiplie par - $\mu d t$, et qu'on prenne la somme de tous ces produits depuis $\approx=0$ jusqu'à $\approx=z_{1}$, il viendra

d'ou lon obtient enfin

$$
\boldsymbol{B}=-2 \pi d t \int_{0}^{z_{1}} \mu \boldsymbol{r} d z
$$

$$
\text { 41. } \quad d q=-2 \pi d t\left(\int_{0}^{r} \frac{d z_{1}}{d t} r d r+\int_{0}^{z_{1}} \mu r d z\right) \text {. }
$$

Puisque $d q$ doit rester le même quel que soit $r$, pourvu qu'il soit $>\boldsymbol{r}^{\prime}$. 
il faut que la différence partielle du second membre de l'équation précedente par rapport à $r$ soit égale à zéro. Celà doune

$$
r \frac{d z_{1}}{d t}+r \mu_{z_{1}} \frac{d z_{1}}{d r}+\int_{0}^{z_{1}} \frac{d(\mu r)}{d r} d z=0
$$

ou bien, si l'on substitue la valeur de $\frac{d(\mu r)}{d r}$ tirée de l'équation (39), et qu'on divise ensuite par $r$,

$$
\text { 42. } \frac{d z_{1}}{d t}+\mu_{z_{1}} \frac{d z_{1}}{d r}-w_{x_{1}}=0,
$$

$\mu_{z_{1}}$ et $w_{z_{1}}$ désignant les valeurs de $\mu$ et $w$ correspondantes $\dot{a} z=z_{1}$. L'équation (42) est en effet celle qu'on obtient, quand on veut exprimer la condition que les molecules de la surface supérieure, dont l'équation est $z-z_{1}=0$, restent toujours dans cette surface, pendant qu'elle varie. Nous voyons, que cette supposition est essentiellement liée à la continuité de la masse liquide, et qu'elle en est une conséquence absolument nécessaire. II faut cependant observer, que l'équation (41) n'ayant lieu que pour $r>\boldsymbol{r}^{\prime}$, l'équation (42) ne subsistera pas non plus, que hors de cette limite de $r$. 